\title{
Role of ischemia-reperfusion in oxidative stress-mediated injury during kidney transplantation
}

\author{
Cristóbal Ávila, Lucas Líbano, Ignacio Rojas and Ramón Rodrigo* \\ Laboratory of Oxidative Stress and Nephrotoxicity, Molecular and Clinical Pharmacology Program, Institute of Biomedical Sciences, Faculty of Medicine, University \\ of Chile, Chile
}

\begin{abstract}
Renal transplant (RT) is the definitive treatment for end-stage renal disease, which is known as a high prevalence pathology with strong economic repercussion both for patients and health systems. Solid organ transplantation is a classically described clinical setting in which massive amounts of reactive oxygen species (ROS) are produced due to ischaemia-reperfusion, thus becoming an essential pathophysiological element involved in delayed graft function in the context of RT. Nevertheless, no clinical protocol yet exists to counteract the damage mediated by ROS intensively produced throughout the transplant process. The available evidence shows a number of successful experiences in the use of antioxidant supplementation and reinforcement over other oxidative stress-related pathologies. This article addresses the pathophysiological role of oxidative stress in RT and its known consequences in function and structure of the allograft, with the objective of gathering consistent information that demonstrates the central role of oxidative stress in this pathology, and to consider it as a possible therapeutic approach in the future.
\end{abstract}

\section{Introduction}

Worldwide, chronic kidney disease (CKD) is a highly prevalent pathology [1]. The main underlying diseases associated with CKD are hypertension and type 2 diabetes mellitus [2], with a prevalence of $27,6 \%$ and $12,3 \%$ respectively, according to data from the 20162017 Chilean National Health Survey [3]. National prevalence for this disease has not yet been determined in Chile, but the estimated global prevalence for CKD is $10 \%$ [4] and currently 1260 patients per million inhabitants are undergoing chronic hemodialysis, compared to 12.7 patients per million in 1980 , according to data from the dialysis registry of the Chilean Nephrology Society [5]. According to estimates from the 2015 Global Burden of Disease study, approximately 1.2 million deaths are attributable to CKD [6], making it plausible to state that kidney disease could contribute more mortality than the main four target diseases defined by the WHO Global Action Plan for the Prevention and Control of Non-Communicable Diseases [7]. Reports from US Renal Data System show that in 2014 there were 120,688 new cases of end-stage renal disease (ESRD, defined as a glomerular filtration rate of less than $15 \mathrm{ml} / \mathrm{min} / 1.73 \mathrm{~m}^{2}$ ) and 678,383 people were treated for this disease, indicating a rise in comparison to 2013 [8]. In addition, ESRD has a high economic impact on healthcare systems: in the US, Medicare cost of this disease has grown 57\% from 1999 to 2004, representing $6,7 \%$ of the total medical expenditures.

CKD ultimately results in kidney failure, which requires treatment by renal replacement therapy (RRT): peritoneal dialysis, hemodialysis or renal transplantation (RT). Amongst them, RT is the gold-standard treatment for patients with ESRD, being the most cost-effective measure compared to other RRT options: US estimates suggest that annual Medicare cost of RT is lower than hemodialysis and peritoneal dialysis $[9,10]$. Cardiovascular event-related mortality is 10 to 30 times higher in patients undergoing hemodialysis than general population [11]. Despite this risk being lower in RT recipients compared to hemodialysis patients, it is still 2 times higher than in healthy patients [12].
Ischaemia-reperfusion (IR) is an inevitable condition during RT, with several studies reporting an association of IR during RT with an impairment in both short and long-term graft survival $[13,14]$. Despite the above, no clinical protocol yet exists to counteract the damage produced by IR throughout the transplant process.

\section{Role of ischaemia/reperfusion in renal transplantation: pathophysiological bases}

Ischaemia-reperfusion injury (IRI) is defined as the damage produced in a tissue subsequently to blood flow restoration (reperfusion), after being previously subjected to limitation of blood flow (ischaemia) for a period of time extended enough to generate hypoxic damage.

Clamping and section of the renal artery during kidney procurement surgery mark the start of renal ischaemia, to be later reperfused after arterial anastomosis in the recipient. Both ischaemia and reperfusion have been demonstrated to be sources of production of reactive oxygen species (ROS), and ultimately producing oxidative stress-mediated damage in the transplanted organ.

Tissue hypoxia generated during ischaemia induces a metabolic shift in the cell from aerobic to anaerobic pathways, which entails a decrease in the generation of adenosine triphosphate (ATP) and an increase in lactate concentrations, leading to intracellular acidosis [15]. ATP depletion causes an electrolytic alteration consisting of an

*Correspondence to: Ramón Rodrigo, Laboratory of Oxidative Stress and Nephrotoxicity, Molecular and Clinical Pharmacology Program, Institute of Biomedical Sciences, Faculty of Medicine, University of Chile, P.O.B. 70058, Chile, E-mail: rrodrigo@med.uchile.cl

Key words: Ischaemia-reperfusion, kidney transplant, oxidative stress, delayed graft function

Received: April 25, 2019; Accepted: May 14, 2019; Published: May 17, 2019 
increase of $\mathrm{Na}^{+}$and water influx, and an intracellular $\mathrm{Ca}^{+2}$ overload [16]. Furthermore, hypoxia reduces the activity of antioxidant enzymes such as superoxide dismutase (SOD), catalase (CAT) and glutathione peroxidase (GSH-Px), and inhibits the expression of cytochrome c oxidase, increasing the production of ROS following an oxygen concentration increase (e.g. during reperfusion) $[17,18]$. The previously described events produce uncoupling of the mitochondrial respiratory chain and weakening of the antioxidant system, allowing a ROS burst during reperfusion, which causes oxidative damage to biomolecules including proteins, lipids and deoxyribonucleic acid (DNA), ending in processes of apoptosis and cell death [15]. In addition, evidence suggests that NADPH oxidase (an enzyme complex that catalyzes the production of a superoxide free radical by transferring one electron from NADPH to oxygen) is activated during the IRI process, having a potential role in the pathophysiology of renal damage in this particular context [19] (Figure 1).

\section{Clinical correlation: ischaemia-reperfusion injury and delayed graft function}

The most frequent post-RT complications are acute kidney injury and acute and chronic graft rejection. The development of these phenomena is multifactorial, including IRI and host/recipient histocompatibility profiles[15,20]. Therefore, many functional parameters in the graft are useful to assess the risk of developing these complications, but one of them has significant clinical relevance because of its frequent incidence in RT and its correlation with long and short-term allograft outcomes [15,21,22]: delayed graft function (DGF), which can be defined as the requirement of at least one dialysis session during the first week post-transplantation [23].

There are different risk factors involved in DGF development, a main one being IRI $[24,25]$. Moreover, several studies confirm the correlation between oxidative stress and DGF development, for example, by linking glutathione S-transferase (antioxidant enzyme) polymorphism GSTM1 and GSTT1 in donors and recipients, to DGF and OS parameters in RT recipients. The results showed that lipoperoxidation, an OS parameter, was significantly higher in patients with DGF and also the frequency of GSTM1 null was significantly higher in patients who developed DGF [26]. Another study Mandegary, et al. investigated the association between $\mathrm{p} 22^{\text {phox }}(\mathrm{C} 242 \mathrm{~T})$, a polymorphic subunit of NADPH-oxidase, which has a critical role in the activation and stabilization of this prooxidant enzyme involved in the production of superoxide, triggering inflammatory injuries to the kidney. The results showed a significant association between $\mathrm{p} 22^{\text {phox }} \mathrm{C} 242 \mathrm{~T}$ polymorphism in the recipient and DGF occurrence [27].

Also, published data La Manna, et al. demonstrated the correlation between low levels of DNA oxidation and apoptosis at 6 months post-RT, with an improved function recovery in kidney allografts, supporting the main role of oxidative stress in graft outcomes [28]. Furthermore, it has been established that malondialdehyde (MDA) levels, a lipoperoxidation and oxidative stress marker, were consistently higher in RT recipient patients that developed DGF compared to those who did not. Also, MDA levels were lower in patients with a better renal function over time. First-day MDA measurements after RT were proven useful as an early marker for DGF[ 29].

\section{Evidence regarding antioxidant supplementation in ischaemia/reperfusion models}

Given the proven association between IRI, oxidative stress and graft outcomes, several authors have investigated the use of antioxidant therapy in RT.

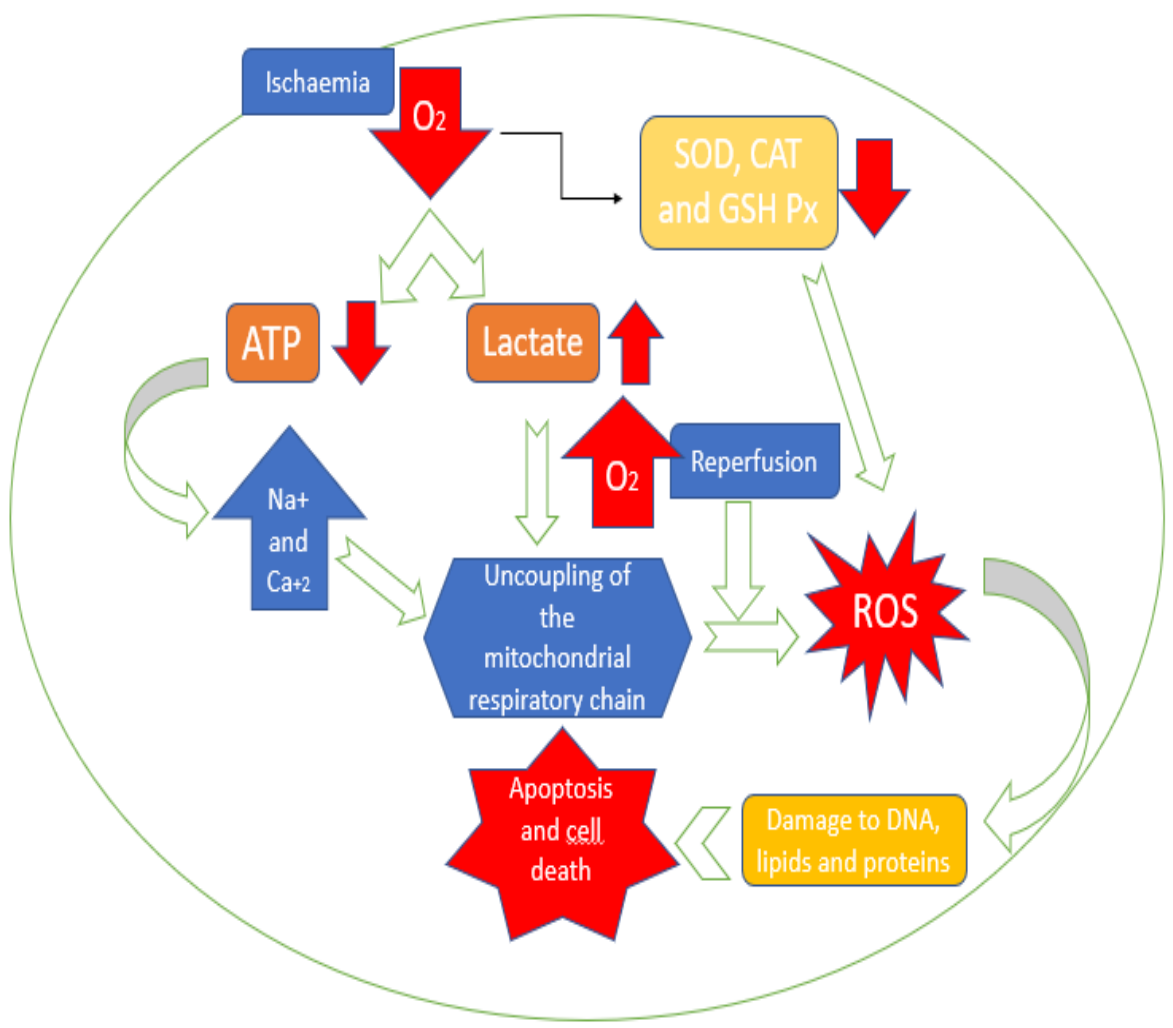

Figure 1. Role of ischemia-reperfusion in the generation of damage mediated by oxidative stress. 
Long, et al. studied the attenuation of IRI in mice kidney by preconditioning with oleanolic acid, a natural triterpenoid present in various food products, like vegetable oils, with a proven antioxidant activity $[30,31]$. The protocol consisted in the distribution of rats into 3 main groups; a sham group, which received sham surgery, an ischaemia-reperfusion group, and an ischaemia-reperfusion group that received an injection of oleanolic acid for 15 consecutives days prior to the induction of renal ischaemia-reperfusion. The renal function assessment was measured by determination of blood urea nitrogen, creatinine, kidney injury molecule-1 (KIM-1) and others. Besides, antioxidant activity was assessed by MDA levels, SOD, CAT and GSH-Px activity. The results demonstrated that preconditioning by oleanolic acid was able to prevent IR-induced renal injury, as evidenced by decreased serum levels of blood urea nitrogen, creatinine and lactate dehydrogenase, and renal levels of KIM-1, compared with the I/R group. Furthermore, said preconditioning exhibited antioxidant effects in IR-oleanolic acid, as reflected by decreased MDA levels, increased SOD, CAT and GSH-Px activities, and increased GSH content compared with the IR rats [32]. Another study of natural antioxidants supplementation is the use of leutoline (Liu, et al.), a flavonid obtained from a variety of plants like carrot, peppers and olive oil that has shown potent antioxidant properties [33]. Similarly, to the previously described, this study split rats into 3 groups, a sham group, an ischaemia-reperfusion group and an ischaemia-reperfusion group supplemented with leutoline. The results reported a significant enhance of renal function biomarkers (i.e. blood urea nitrogen and creatinine levels) and antioxidant enzyme function (e.g. CAT, SOD and GSH-Px) in the supplemented group compared to the ischaemia-reperfusion group [34].

Other studies were focused on the use of non-polyphenolic substances, such as Cusmano, et al. who evaluated the effect of atorvastatin (ATOR) and N-acetylcysteine (NAC) in the prevention of IRI on a murine model. Both substances possess anti-inflammatory, antiapoptotic and antioxidant properties. Mice were divided into 4 groups: control, NAC, ATOR and NAC+ATOR. Myeloperoxidase (MPO), SOD, CAT and GSH-Px levels were measured in renal tissue, plus the assessment of microstructural alterations (glomerulopathy, interstitial infiltrate, tubular anomalies and vasculopathy). GSHPx activity levels were significantly higher and MPO activity levels were significantly lower in the three treated groups compared to control group, in addition to a lower ischaemic tubular injury index in NAC+ATOR group compared to placebo [35]. Another research analyzed the therapeutic effects of NAC in deceased donor RT, related to oxidative stress (Danilovic, et al.). Variables measured included creatininemia, GFR estimated by MDRD and Cockcroft-Gault methods (eGFR), the incidence of DGF and the analysis of reactive substances of thiobarbituric acid (TBARS), an oxidative stress biomarker. Two groups were established: NAC and placebo. NAC treated group showed lower creatininemia and higher GFR estimation during the first 90 days post-transplantation and a year after RT, compared to control group. Furthermore, rate DGF and TBARS levels were significantly lower in NAC treated group compared to placebo [36].

\section{Conclusions}

The available evidence allows us to conclude that during RT, inevitable damage is produced by ischaemia-reperfusion, which involves a decrease in antioxidant enzymes activity and enhanced ROS production resulting in cell death and the incidence of negative graft outcomes. Furthermore, there is a correlation between IRI, oxidative stress parameters and DGF, evidenced both in clinical and pre-clinical antioxidant supplementation models. Nevertheless, more solid studies are necessary to prove the efficacy of antioxidant supplementation in RT and to perfect a therapeutic strategy. Finally, an approach to $\mathrm{RT}$ management and care under the oxidative stress paradigm is an exciting, new path that has yet to be fully understood, but could potentially offer novel, safe and cost-effective treatments aimed to obtain optimized graft outcomes.

\section{References}

1. Romagnani P, Remuzzi G, Glassock R (2017) Chronic kidney disease. Nat Rev Dis Primers 3: 17088. [Crossref]

2. Hill NR, Fatoba ST, Oke JL, Hirst JA, O'Callaghan CA, et al. (2016) Global Prevalence of Chronic Kidney Disease - A Systematic Review and Meta-Analysis. PLoS One 11: e0158765. [Crossref]

3. Margozzini P, Passi Á (2018) Encuesta Nacional de Salud, ENS 2016-2017: un aporte a la planificación sanitaria y políticas públicas en Chile. ARS MEDICA Revista de Ciencias Médicas p. 30.

4. Coresh J, Byrd-Holt D, Astor BC, Briggs JP, Eggers PW, et al. (2005) Chronic Kidney Disease Awareness, Prevalence, and Trends among U.S. Adults, 1999 to 2000. J Am Soc Nephrol pp. 180-188.

5. Dialysis Registry (2018) Chilean Society of Nephrology, Valparaíso.

6. GBD 2015 Mortality and Causes of Death Collaborators (2016) Global, regional, and national life expectancy, all-cause mortality, and cause-specific mortality for 249 causes of death, 1980-2015: a systematic analysis for the Global Burden of Disease Study 2015. Lancet 388: 1459-1544. [Crossref]

7. Crews DC, Bello AK, Saadi G, World Kidney Day Steering Committee (2019) Burden, Access, and Disparities in Kidney Disease. Indian J Nephrol 29: 77-83.

8. Saran R, Robinson B, Abbott KC, Agodoa LYC, Albertus P, et al. (2017) US Renal Data System 2016 Annual Data Report: Epidemiology of Kidney Disease in the United States. Am J Kidney Dis 69: A7-A8.

9. Foley RN, Collins AJ (2007) End-stage renal disease in the United States: an update from the United States Renal Data System. J Am Soc Nephrol 18: 2644-2648. [Crossref]

10. Ferguson TW, Tangri N, Rigatto C, Komenda P (2015) Cost-effective treatment modalities for reducing morbidity associated with chronic kidney disease. Expert Rev Pharmacoecon Outcomes Res 15: 243-252.

11. Foley RN, Parfrey PS, Sarnak MJ (1998) Clinical epidemiology of cardiovascular disease in chronic renal disease. Am J Kidney Dis pp. S112-S119.

12. Sarnak MJ, Levey AS, Schoolwerth AC, Coresh J, Culleton B, et al. (2003) Kidney disease as a risk factor for development of cardiovascular disease: a statement from the American Heart Association Councils on Kidney in Cardiovascular Disease, High Blood Pressure Research, Clinical Cardiology, and Epidemiology and Prevention. Circulation 108: 2154-2169.

13. Yarlagadda SG, Coca SG, Formica RN Jr, Poggio ED, Parikh CR (2009) Association between delayed graft function and allograft and patient survival: a systematic review and meta-analysis. Nephrol Dial Transplant 24: 1039-1047.

14. Gueler F, Gwinner W, Schwarz A, Haller H (2004) Long-term effects of acute ischemia and reperfusion injury. Kidney Int 66: 523-527. [Crossref]

15. Sotomayor CG, Cortés I, Gormaz JG, Vera S, Libuy M, et al. (2017) Role of Oxidative Stress in Renal Transplantation: Bases for an n-3 PUFA Strategy Against Delayed Graft Function. Curr Med Chem 24: 1469-1485.

16. Kosieradzki M, Rowinski W (2008) Ischemia/reperfusion injury in kidney transplantation: mechanisms and prevention. Transplant Proc 40: 3279-3288.

17. Li C, Jackson RM (2002) Reactive species mechanisms of cellular hypoxiareoxygenation injury. Am J Physiol C227-C241.

18. Bayrak O, Bavbek N, Karatas OF, Bayrak R, Catal F, et al. (2008) Nigella sativa protects against ischaemia/reperfusion injury in rat kidneys. Nephrol Dial Transplant pp. 2206-2212.

19. Tabriziani H, Lipkowitz MS, Vuong N (2018) Chronic kidney disease, kidney transplantation and oxidative stress: a new look to successful kidney transplantation. Clin Kidney J 11: 130-135.

20. Saat TC, van den Akker EK, IJzermans JNM, Dor FJMF, de Bruin RWF (2016) Improving the outcome of kidney transplantation by ameliorating renal ischemia reperfusion injury: lost in translation? J Transl Med 14: 20. 
21. Ponticelli C (2014) Ischaemia-reperfusion injury: a major protagonist in kidney transplantation. Nephrol Dial Transplant pp. 1134-1140.

22. Tapiawala SN, Tinckam KJ, Cardella CJ, Schiff J, Cattran DC, et al. (2010) Delayed graft function and the risk for death with a functioning graft. J Am Soc Nephrol 21: 153-161. [Crossref]

23. Yarlagadda SG, Coca SG, Garg AX, Doshi M, Poggio E, et al. (2008) Marked variation in the definition and diagnosis of delayed graft function: a systematic review. Nephrol Dial Transplant 23: 2995-3003. [Crossref]

24. Kumar A, Hammad A, Sharma AK, Mc-Cardle F, Rustom R, et al. (2015) Oxidative stress in kidney transplant biopsies. Exp Clin Transplant 13 Suppl 1: 207-213. [Crossref]

25. Perico N, Cattaneo D, Sayegh MH, Remuzzi G (2004) Delayed graft function in kidney transplantation. Lancet 364: 1814-1827. [Crossref]

26. Azmandian J, Mandegary A, Pootari M, Nematolahi MH, Ebadzadeh MR, et al. (2017) Role of Donors and Recipients' Glutathione S-Transferase Gene Polymorphisms in Association of Oxidative Stress With Delayed Graft Function in Kidney Allograft Recipients. Iran J Kidney Dis 11: 241-248.

27. Mandegary A, Rahmanian-Koshkaki S, Mohammadifar MA, Pourgholi L, Mehdipour $\mathrm{M}$, et al. (2015) Investigation of association between donors' and recipients' NADPH oxidase p22(phox) C242T polymorphism and acute rejection, delayed graft function and blood pressure in renal allograft recipients. Transpl Immunol 32: 46-50.

28. La Manna G, Lanci N, Della Bella E, Comai G, Cappuccilli ML, et al. (2011) Reduction of oxidative damage reflects a better kidney transplantation outcome. Am J Nephrol 34: 496-504.
29. Fonseca I, Reguengo H, Almeida M, Dias L, Martins LS, et al. (2014) Oxidative stress in kidney transplantation: malondialdehyde is an early predictive marker of graft dysfunction. Transplantation 97: 1058-1065.

30. Wang X, Ye XL, Liu R, Chen HL, Bai H, et al. (2010) Antioxidant activities of oleanolic acid in vitro: possible role of Nrf2 and MAP kinases. Chem Biol Interact 184: 328-337.

31. Liu J, Wang X, Liu R, Liu Y, Zhang T, et al. (2014) Oleanolic acid co-administration alleviates ethanol-induced hepatic injury via $\mathrm{Nrf}-2$ and ethanol-metabolizing modulating in rats. Chem Biol Interact 221: 88-98.

32. Long C, Yang J, Yang H, Li X, Wang G (2016) Attenuation of renal ischemia/reperfusion injury by oleanolic acid preconditioning via its antioxidant, antiâ€'inflammatory, and antiâ€'apoptotic activities. Mol Med Rep 13: 4697-4704. [Crossref]

33. Xin SB, Yan H, Ma J, Sun Q, Shen L (2016) Protective Effects of Luteolin on Lipopolysaccharide-Induced Acute Renal Injury in Mice. Med Sci Monit pp. 5173-5180.

34. Liu Y, Shi B, Li Y, Zhang H (2017) Protective Effect of Luteolin Against Rena Ischemia/Reperfusion Injury via Modulation of Pro-Inflammatory Cytokines, Oxidative Stress and Apoptosis for Possible Benefit in Kidney Transplant. Med Sci Monit 23: 5720-5727.

35. Cusumano G, Romagnoli J, Liuzzo G, Ciavarella LP, Severino A, et al. (2015) $\mathrm{N}-$ Acetylcysteine and High-Dose Atorvastatin Reduce Oxidative Stress in an IschemiaReperfusion Model in the Rat Kidney. Transplant Proc pp. 2757-2762.

36. Danilovic A, Lucon AM, Srougi M, Shimizu MHM, Ianhez LE, et al. (2011) Protective effect of $\mathrm{N}$-acetylcysteine on early outcomes of deceased renal transplantation. Transplant Proc 43: 1443-1449.

Copyright: (C2019 Ávila C. This is an open-access article distributed under the terms of the Creative Commons Attribution License, which permits unrestricted use, distribution, and reproduction in any medium, provided the original author and source are credited. 